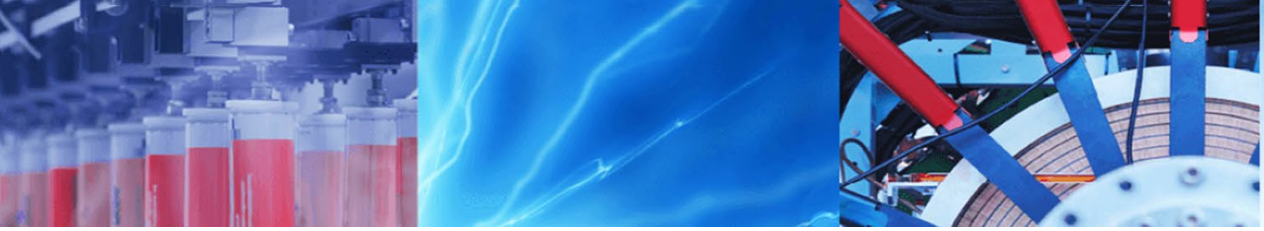

Research Article

\title{
Highly accurate space-time coupled least-squares finite element framework in studying wave propagation
}

\author{
M. A. Saffarian ${ }^{1}$ A. R. Ahmadi ${ }^{2}$ M. H. Bagheripour ${ }^{1}$
}

Received: 15 October 2019 / Accepted: 10 March 2020 / Published online: 16 March 2020

(c) Springer Nature Switzerland AG 2020

\begin{abstract}
Simulation of stress wave propagation through solid medium is commonly carried using Galerkin weak-form cast over decoupled space and time domains. In this paper, accuracy of this commonly utilized framework is compared to that of the variationally-consistent least-squares form of the wave equation cast over space-time domain. The two formulations are tested for numerical dispersion and numerical diffusion, through two test cases. The first case studies the dispersion in harmonic shear wave propagation through a soil column over a wide range of forcing frequencies. The second test case investigates numerical diffusion in an axial wave propagation generated by constant force; which is removed after a certain time to allow free vibration to take place. Low numerical dispersion and numerical diffusion as well as high rates of convergence are the main advantages of the coupled least-squares (CLS) computational framework; when compared to the decoupled Galerkin (DG) framework. Based on studies presented here, CLS has low dispersion; yielding errors with one to two orders of magnitude less than that of DG. Also, the numerical diffusion present in DG framework causes a $\% 40$ error in DG's prediction of the stress-wave intensity. Furthermore, accumulative error during evolution is virtually nonexistent for CLS, whereas, the error steadily increases as the solution evolves in DG framework. It is also demonstrated that CLS feature of temporal meshing allows for faster computations.
\end{abstract}

Keywords Wave equation · Shear wave propagation - Impact wave propagation · Space-time coupled · Least squares · Higher continuity hierarchical finite elements · Numerical diffusion · Numerical dispersion · Variational-consistency

\section{Introduction}

Simulation of stress wave propagation through solid medium is an active area of research in many engineering fields; for which, numerically based computational methods are employed extensively. In particular, finite elements method has been successfully utilized in handling serious computational issues; such as, complex boundary conditions, geometric irregularities, and non-homogeneity of the medium, Kramer [1].

Computational methods employed in studying wave propagation, fall under two broad categories of (a)
Time domain, which is the most general computational approach; and (b) Hybrid Frequency-time domain methods, which are viable for linear or pseudo-linear problems. In all these studies, general computational techniques of Finite Difference, Boundary Elements, and Finite Elements along with its Isogeometric and Meshless variations, have been widely used [2-5]. Transfer Function Method is a frequency based technique; which has also been used to conduct such studies, Mirzaei, Lisha and Shojaei et al. [4, 6, 7]. Saffarian and Bagheripour $[8,9]$ investigated the earthquake response of surcharged soil layers using Hybrid Frequency Time Domain (HFTD) concept. They used transfer

\footnotetext{
$\triangle$ A. R. Ahmadi, a.ahmadi@kgut.ac.ir; M. A. Saffarian, amin.saffarian@eng.uk.ac.ir; M. H. Bagheripour, bagheri@uk.ac.ir|' Civil Engineering Department, Shahid Bahonar University of Kerman, Kerman, Iran. ${ }^{2}$ Department of Mechanical Engineering, Kerman Graduate University of Technology, Kerman, Iran.
} 
function technique to solve the problem and to develop a solution algorithm.

Sun and Chung [10] in their evaluation of the mathematical models used in study of ground response to seismic waves, investigated a horizontally layered soil profile using 1D and 2D solution methods; and concluded that the two sets of results were comparable with negligible differences. In studies conducted here, a one dimensional soil layer model is adopted.

Semi-Discrete finite element is the common computational framework utilized in development of commercially available software packages. In this framework, partial differential equations defined over a space-time domain are converted into ordinary differential equations in time; that are solved using direct numerical integration techniques, such as Newmark or Wilson- $\theta$ methods. These methods yield unsatisfactory results when high-frequency excitation is induced; as demonstrated by Hulbert and Hughes [5] in studying the problem of an elastic axial bar hitting a wall.

Various improvements on semi-discrete methodologies have been introduced, Hilber and Hughes [11-15]. Even though the semi-discrete finite elements approach has led to significant improvements in simulation of elastic wave propagation, problems with high frequency loading and sharp temporal gradients still present a significant challenge.

Keeping the space-time coupled during the computational process has successfully been used in study of transient problems. In this approach finite element interpolation is performed over spatial and temporal dimensions, simultaneously. Argyris and Scharpf [16] were the first to carry studies over the coupled domain. Further research efforts by Argyris, Fried, Zienkiewicz, Wilson, and Oden investigate computational aspects of time dependent processes within the finite element context [17-22]. The space-time coupled finite element processes have evolved into two categories of time-continuous and time-discontinuous methodologies. Many researchers have investigated the merits of the time-continuous space-time finite elements [23-30]. Discontinuous finite elements, first introduced by Reed and Hill [31], were applied successfully by Hughes and Hulbert [32] in study of elastic wave propagation. Their work has demonstrated that the Galerkin form of the wave equation can yield accurate and stable results when time-discontinuous space-time elements are used in conjunction with least squares stabilizers. It should be noted that numerical stabilizers are usually problem dependent, Guo et al. and Izdpanah et al. [33, 34].

Bell and Surana in [35] demonstrated that space-time coupled least squares formulation of fluid dynamic equations can yield a stable evolutionary process with highly accurate results; without a need for numerical stabilizers.
In their studies, since high order Lagrange-type polynomials lead to ill conditioned coefficient matrices, $p$-version hierarchical finite elements, first introduced by Szabo [36], were employed. Surana et al. [37] further developed the $p$-version hierarchical elements with higher global continuity to be used for a variety of scientific studies. Higher continuity finite elements were further improved upon, to maintain arbitrary levels of global continuity for quadrilateral elements, Ahmadi et al. [39].

A study of Galerkin and least-squares forms in higher continuity spaces was performed by Ahmadi [38]. It was shown that casting the computational process in higher continuity spaces, not only has the advantage of formulating strong form of the equations, it can also lead to higher rates of convergence and more stable computational processes. In a series of papers by Surana, Ahmadi, and Reddy [40-42] a variationally-consistent computational framework based on higher global continuity, was introduced. Variational consistency ensures that the highest order differential exists in the solution space searched by the weak form.

In this paper, the one-dimensional wave equation is formulated and studied within a variationally-consistent framework. Its computational merits are compared to the commonly utilized Galerkin formulation; available in almost any finite element package. Comparative studies reveal that the variationally-consistent least squares form cast over a coupled space-time domain yields a highly time-accurate computational process without the need for any type of numerical stabilizer.

To investigate the accuracy and robustness of spacetime coupled least squares finite element computational framework, as applied to wave equation, two test cases are considered. The first test is designed to investigate numerical dispersion. It is a one dimensional soil column, based on Sun and Chung [10] model, subjected to harmonic shearing loads running at frequencies that are dominant in seismic waves; i.e. $0.1-100 \mathrm{~Hz}$. In the second test-case, a constant axial force is applied for a period of time and then removed to allow for free vibration to take place. Using extensive computational resources, error due to dispersion was forced down; hence allowing for numerical diffusion, which is an inherent characteristic of the formulation, to become more resolute during the free vibration.

In these studies error in strain wave at the fixed boundary is measured under forced and free vibrating conditions. Errors were also measured at domain's mid-point. Studies herein indicate that the variationally-consistent formulation of least squares cast over space-time domain is a highly accurate and stable, almost no numerical dispersion or diffusion. Furthermore, CLS framework does not require the use of problem-dependent stabilizers, as proposed by Hulbert and Hughes [5]. 
The remainder of this manuscript is organized as follows; the mathematical model describing wave propagation is stated first followed by weak formulations of the said model cast in decoupled Galerkin and coupled least squares forms. The succeeding section contains a series of test cases designed to investigate convergence rates, numerical dispersion, and numerical diffusion for each of the weak forms. At the end, findings are discussed and concluding remarks are stated.

\section{Wave propagation model}

One dimensional linear wave equation can be used to model the axial or shear wave propagation through a medium, i.e.

$\frac{\partial^{2} u}{\partial t^{2}}=c^{2} \frac{\partial^{2} u}{\partial z^{2}}$

where $c=\sqrt{\frac{\lambda}{\rho}}$ is the speed of sound through a medium with elastic modulus of $\lambda$ and mass density of $\rho$; and $u=u(z, t)$ denotes the displacement field in space: $z \in \Omega=[0, H]$ and time: $t \in \mathcal{I}=[0, T]$. The wave equation is supplemented with a set of boundary and initial conditions forming the strong formulation; solution of which is referred to as the strong solution, or exact.

Numerical solution of (1) can be carried in one of two ways; (1) space-time decoupled, or, (2) space-time coupled. In decoupled strategy, Galerkin weak form of (1) is formed over the spatial domain $\Omega$; yielding a set of time dependent differential equations that are solved simultaneously, using finite difference techniques. In the coupled framework, weak form of (1) is cast over the space-time domain. Both formulation strategies are discussed next.

\subsection{Decoupled Galerkin formulation}

Assuming that $c$ is constant, the decoupled Galerkin weak form of (1) can be formed by letting $u(z, t)=\mathbf{N}(z) . \Phi(t)$; thus yielding, ${ }^{1}$

$\int_{\Omega}\left(\mathbf{N}^{T} \cdot \mathbf{N}\right) d z \frac{\partial^{2} \mathbf{\Phi}}{\partial t^{2}}+c^{2} \int_{\Omega}\left(\frac{d \mathbf{N}^{T}}{d z} \cdot \frac{d \mathbf{N}}{d z}\right) d z \mathbf{\Phi}=0$

or, $\mathbf{M} \ddot{\boldsymbol{\Phi}}+\mathbf{K} \boldsymbol{\Phi}=\mathbf{0}$ where $\mathbf{M}$ and $\mathbf{K}$ are respectively the distributed mass and stiffness matrices of the medium under consideration. The resulting set of ordinary differential equations can be solved by a number of methods, e.g. Newmark, Wilson- $\theta$ amongst others. All of these evolutionary schemes are finite difference based and they all

\footnotetext{
1 Bold face symbols are used to indicate matrices and vectors.
}

use stabilizing parameters that may depend on physical and/or loading features of the problem being solved.

\subsection{Coupled formulations}

In development of coupled formulation, the Galerkin weak form can be constructed over the entire space-time domain, i.e.

$\int_{\mathcal{I}} \int_{\Omega}\left(\frac{\partial \mathbf{N}^{T}}{\partial t} \frac{\partial \mathbf{N}}{\partial t}-c^{2} \frac{\partial \mathbf{N}^{T}}{\partial z} \frac{\partial \mathbf{N}}{\partial z}\right) d z d t \cdot \Phi=\mathbf{0}$

The decoupled and the coupled Galerkin forms given by (2) and (3), both require global continuity of the approximating function, i.e. $u \in \mathbb{C}^{0}$. These forms are variationally-inconsistent, since the highest derivative of $u$, as stated by (1), does not exist in the solution space. Hence, the resulting system of equations does not yield a stable evolution; thus requiring numerical stabilizers. Hughes et al. [32] have developed suitable space-time evolutionary schemes based on least squares stabilizers that would yield convergent solutions.

As mentioned earlier, present work focuses on least squares formulation of the problem; which yields a variationally-consistent process without the need for numerical stabilizers. Assuming $u(z, t)=\mathbf{N}(z, t) \cdot \boldsymbol{\Phi}$ the residual function will be $R(u)=\frac{\partial u^{2}}{\partial t^{2}}-c^{2} \frac{\partial u^{2}}{\partial z^{2}}$. Least squares weak form of (1) can be constructed be letting the weight function to be defined as variation of the residual function $R(u)$, resulting,

$\int_{\mathcal{I}} \int_{\Omega} \mathbf{Q}^{T} \cdot \mathbf{Q} d z d t \cdot \boldsymbol{\Phi}=\mathbf{0}$

where $\mathbf{Q}=\frac{\partial^{2} \mathbf{N}}{\partial t^{2}}-c^{2} \frac{\partial^{2} \mathbf{N}}{\partial z^{2}}$. The least squares weak form given in (4) requires global continuity of the approximation function as well as its first derivative in both space and time. This is a variationally-consistent form since the second derivative exists in the solution space. Furthermore, existence of high gradients in the solution space would need high-order approximating polynomials to yield stable and accurate results. Hence, $\mathbb{C}^{1}$ hierarchical finite elements as developed by Surana et al. [37] are utilized herein.

\section{Numerical studies}

Two case studies are considered herein; the first is a soil column subjected to harmonic shear waves and the second simulates axial wave propagation in a rod under constant and harmonic loading cases. The first case study measures error due to numerical dispersion as well as accumulative evolution error. The second test case is designed to measure error due to numerical diffusion. 
In this case, dispersion error is minimized through highly refined temporal meshing, hence allowing for numerical diffusion to dominate the error measure. All studies compare accuracy and convergence characteristics of CLS and DG.

\subsection{Space-time interpolation}

The time domain of interest, $\mathcal{T}_{\mathcal{D}}=[0, T]$ is discretized as $\mathcal{T}=\left\{t_{0}, t_{1}, \ldots, t_{m}\right\}$. Noting problem's evolutionary nature, computations can be performed in a successive manner over a set of $m$ space-time domains: $\left\{\Omega \times \mathcal{I}_{i}\right\}_{i=1, m}$ instead of the entire space-time domain of $\Omega \times \mathcal{I}$; thus significantly reducing the computational cost. In this process, space-time slabs $\Omega \times \mathcal{I}_{i}=[0, H] \times\left[t_{i-1}, t_{i}\right]$ are meshed and analyzed progressively. In all computations, in order to maintain numerical accuracy and evolution stability, for any element " $e$ " of size $\left(\Delta z_{e} \times \Delta t_{e}\right)$, element speed, $v_{e}$, is selected to be larger than the wave speed, $c$, i.e.

$v_{e}=\frac{\Delta z_{e}}{\Delta t_{e}} \geq c=\sqrt{\frac{\lambda}{\rho}}$

\subsubsection{Time slab meshing}

In the space-time coupled framework, computations are successively carried out over time increments $\mathcal{I}_{i}=\left[t_{i-1}, t_{i}\right]$ . If the increment $\mathcal{I}_{i}$ is discretized into only one element in $t$-direction, analogous to the decoupled method, then time increment's size must be selected such that (5) would remain valid. However, if the slab is discretized in time in such a way that (5) would hold true over each element of the space-time slab, then, it is possible to compute over time increments of longer duration.

\subsection{Measuring error}

In measuring the error in approximate solutions, results are compared to exact values as follows. Considering the approximate and exact curves for a function $u$ over time, average difference and average exact values, over a time segment $\Delta_{i}$, are defined as $D_{i}=\Delta_{i}\left(d_{i}+d_{i+1}\right) / 2$ and $E_{i}=\Delta_{i}\left(e_{i}+e_{i+1}\right) / 2$ where $d_{k}=\left|a_{k}-e_{k}\right|$ in which $a_{k}$ is the approximate value and $e_{k}$ is the exact value at $k$ th time point. The error can be measured using,

$\%$ Error $=100 \times \frac{\sum_{i} D_{i}}{\sum_{i} E_{i}}$

Error due to numerical damping is investigated in the second test case. The error is computed by comparing the area under a half cycle at different time points.

\subsection{Harmonic shear wave loading}

The soil column modeled here has a height of $H=10 \mathrm{~m}$ with elastic modulus of $\lambda=G=7.5 \mathrm{MPa}$ and weight density of $\rho g=20 \mathrm{kN} / \mathrm{m}^{3}$; where $g=9.81 \mathrm{~m} / \mathrm{s}^{2}$. The column is assumed to be initially at rest, before getting excited at its base by a harmonic shear wave of a particular frequency. Hence, the boundary and initial conditions can be written as,

$$
\begin{cases}\text { B.C. } & \\ & u(0, t)=A \sin (\omega t) \\ & u(H, t)=\frac{\partial u}{\partial z}(H, t)=0 \\ \text { I.C. } & \\ & u(z, 0)=\frac{\partial u}{\partial t}(z, 0)=0\end{cases}
$$

where the displacement condition $u(0, t)$ has an amplitude of $A=0.01 \mathrm{~m}$ and frequency $\omega=2 \pi f$, with $f$ selected from the set $\{0.1,0.5,1.5,5,7,10,50,100\}$, defining the test cases examined. Furthermore, since $\mathbb{C}^{1}$ finite elements are employed in studying (4), the stress free boundary condition $\left.\frac{\partial u}{\partial z}\right|_{(z=H, t)}=0$ and zero velocity initial condition are imposed explicitly; a feature that is not available in either of the $\mathbb{C}^{0}$ Galrekin forms given in (2) and (3).

\subsubsection{Exact solution}

A closed form solution of (1) subjected to (7) may be found through the separation of variables technique, yielding,

$u(z, t)=\sum_{\lambda} \frac{16 P_{\lambda} A H \omega \sin ^{2}\left(\frac{\lambda}{4}\right)}{c^{2} \lambda^{3}-4 \lambda \omega^{2} H^{2}}+A \sin (\omega t)$

where $P_{\lambda}=\sin \left(\frac{\lambda z}{2 H}\right)\left(2 H \omega \sin (\omega t)-c \lambda \sin \left(\frac{c \lambda t}{2 H}\right)\right)$ and $\lambda=k \pi, k=1,3, \ldots b$, with $\omega=2 \pi f$. In each test case, the closed form solution is computed by summing the first 5000 terms. All numerical errors are measured with respect to this "exact" distribution of $u$.

\subsubsection{Modeling data}

The decoupled Galerkin form given in (2) is solved for conditions (7) by first using more than 900 plane strain finite elements to form mass and stiffness matrices, and then solving the resulting set of time-dependent ordinary differential equations using Newmark method with stabilizer factors $\alpha=0.3$ and $\beta=0.6$. The results are compared to a uniform mesh of $20 \mathbb{C}^{1}$ space-time finite elements at $p=8$. 


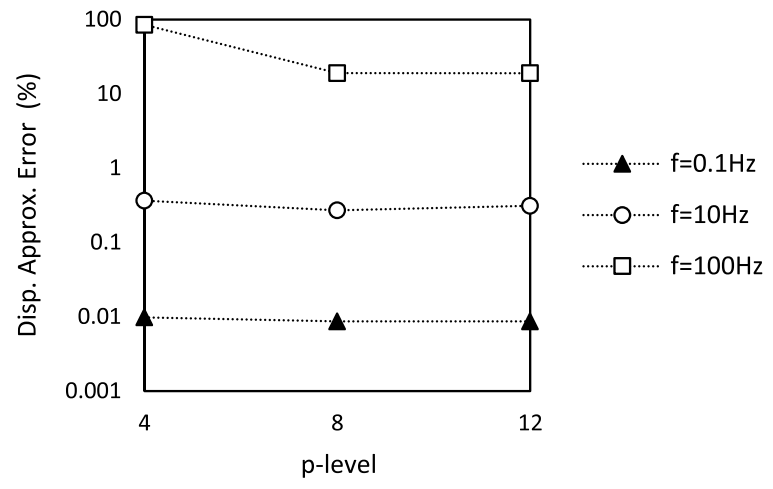

(a) $p$-convergence of displacement

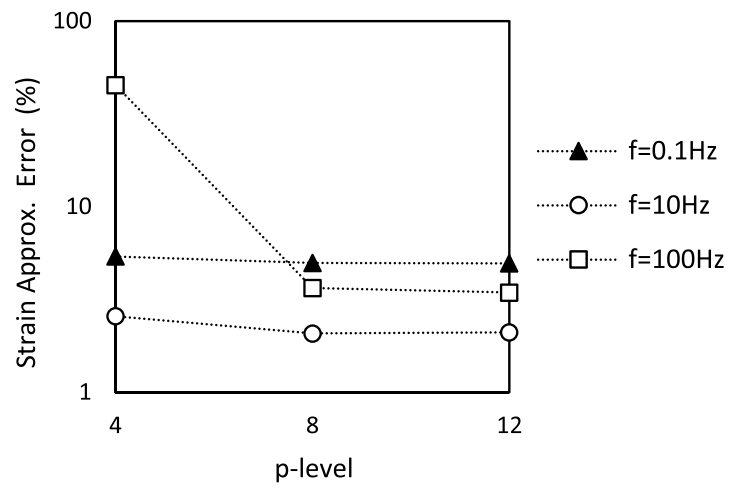

(b) $p$-convergence of strain

Fig. 1 Error in displacement and strain distributions for different order of interpolating polynomial

The two models have about the same number of degrees of freedom.

\subsection{3 $h$ - and $p$-convergence}

As the order of interpolating polynomial, $p$-level, is increased in both $z$ and $t$ directions, one expects a decrease in error; as measured by comparing the computed solution to the exact as given in (8). Using the 20-element uniform mesh, errors in displacement and strain distributions are measured at three frequencies of $\{0.1,10,100\} \mathrm{Hz}$ for $p$-levels of $\{4,8,12\}$, Fig. 1 .

It can be seen from Fig. 1 that except for $f=100 \mathrm{~Hz}$ case, the $p$-level increase is ineffective in reducing computational error. Even for $f=100 \mathrm{~Hz}$ case, the uniform mesh model does not show improvement for $p>8$; thus, indicating that mesh refinement is needed. The $h$-refinement may be accomplished uniformly, leading to larger systems of equations to be solved. However, noting that propagation patterns are most complex close to domain boundaries, results from the 20-element uniform model can be improved upon if smaller-sized elements are placed in vicinity of the boundaries.

\subsubsection{Temporal meshing}

An increase in time increment's length means a decrease in the number of slabs required to reach $T$; however, this does not necessarily mean a faster computational process. Computational cost was measured for three cases of (a) 20 elements at $p=8$, (b) 40 elements at $p=8$ and (c) 20 elements at $p=12$ for cases with time increments that are $\{1,2,4,8\}$-times the $\Delta t$ as required by (5).

As can be seen from Fig. 2, one element in time per slab is not the best option; whereas using two time elements seems to be always better regardless of the increase in $p$-level or the number of spatial elements. Furthermore, in

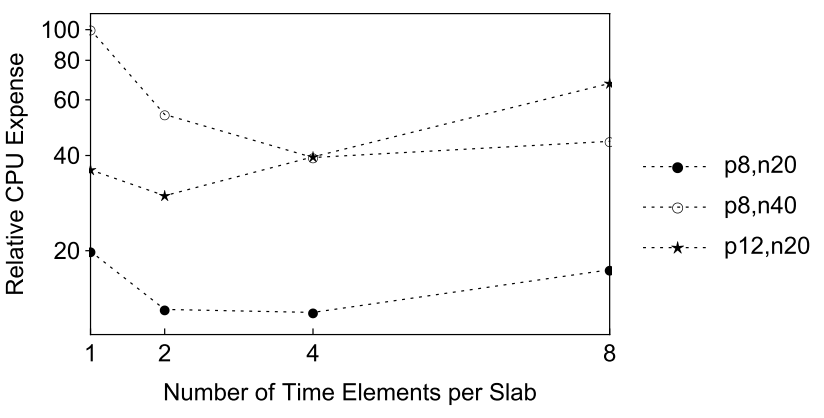

Fig. 2 Relative CPU expense versus number of elements used in a time slab

Table 1 Uniform and nonuniform details of a 20-element mesh

\begin{tabular}{lllll}
\hline & Uniform & I & II & III \\
\hline grade(G) & 1 & 2 & 1.41 & 1.19 \\
\#G-elms. & - & 5 & 7 & 6 \\
elm.MinSize & 0.5 & 0.08 & 0.1 & 0.25 \\
elm.MaxSize & 0.5 & 1.29 & 0.8 & 0.624 \\
G-regionSize & 0.5 & 0.5 & 0.83 & 0.625 \\
\hline
\end{tabular}

case of the lower $p$-level, 4 elements per slab yields even lower computational cost. Note that test cases are set such that the computed results are identical.

\subsubsection{Spatial mesh-grading}

Presence of high gradients in the displacement field, in response to forcing frequency of $100 \mathrm{~Hz}$, necessitates high computational precision for a stable and convergent evolutionary process; hence, $f=100 \mathrm{~Hz}$ case is selected in study of mesh-grading effects on computational accuracy.

Since the results from 20-element uniform-mesh show no improvement for $p$-levels greater than 8 , Fig. 1 , three 


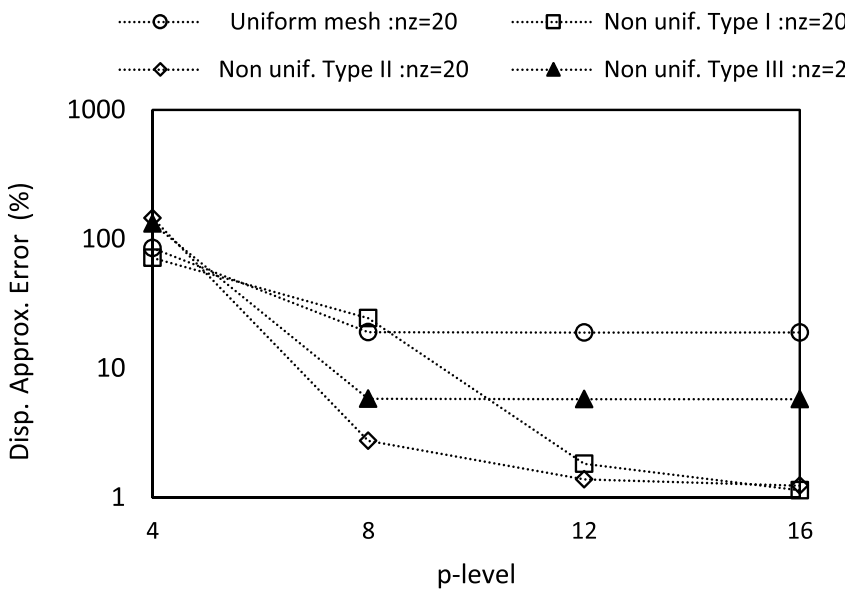

(a) $p$-convergence of displacement

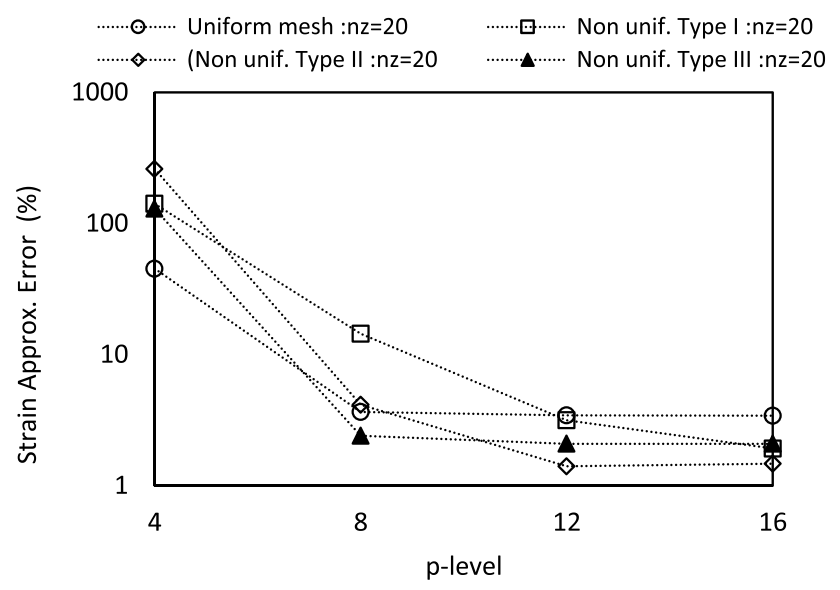

(b) $p$-convergence of strain

Fig. 3 Error in displacement and strain distributions for different order of interpolating polynomial and mesh grade, case: $100 \mathrm{~Hz}$

20-element nonuniform models were developed and tested to obtain improved convergence with almost the same computational cost as the uniform case, Table 1.

Within a region of the domain next to either boundary, certain number of elements change size according to a given growth rate, Table 1; with smallest being located at the boundary. Type-I has the smallest and Type-III has the largest sized elements at domain boundaries. It can be seen from Fig. 3 that only when graded mesh is used, $p$-level increase becomes effective.

Graded mesh Type-ll yields the lowest error for strain and displacement distributions. Type-I which contains the smallest sized elements at domain boundaries, performs poorly at $p=8$ due to existence of large sized element at the end of its growth zone. However, as $p$-level is increased, solution away from the boundary is resolved quickly; and because of the reduced element size, further $p$-level increase affects the error in elements close to boundaries, thus reducing the overall error at a high rate.

In the following section, where the coupled least squares is compared to the decoupled Galerkin, uniform mesh at $p=8$ is used in coupled computations. The decoupled method is analyzed using linear plane-strain elements with characteristic length of about $h=0.01 \mathrm{~m}$ and time increments that are one to two orders of magnitude smaller than the limit set in (5).

\subsubsection{Error over the frequency range}

Percentage of error in displacement and strain computed by Decoupled Galerkin (DG) and Coupled Least Squares (CLS) are graphically compared in Fig. 4. As can be seen from these error graphs, CLS consistently yields more accurate results than DG. Errors in computed displacements are one to two orders of magnitude higher for DG than CLS; and strain errors are close to an order of magnitude higher in DG than CLS.

An interesting point to note is the unexpected reduction in error at $f=1.5$ and $f=50$. The dip is more pronounced for CLS than it is for DG. Reason for this inconsistency is the close proximity of these forcing frequencies to two of the medium's natural frequencies, [1] which are $f_{n}=\frac{c(2 n+1)}{4 H} \quad: \quad n=0,1, \ldots m$. Soil's response at forcing frequencies close to medium's natural ones, is a beating motion. It is a simple motion, oscillating at the induced frequency, with increasing amplitude that would decrease and repeat the pattern over long time-periods. Response to forcing frequencies not close to natural frequencies, is a complex motion produced through wave interactions taking place over short time intervals.

For the problem at hand, the fundamental frequency is $f_{0}=1.516 \mathrm{~Hz}$ which is a simple node-less motion that gets amplified and reduced over long periods of time, for the forcing frequency $f=1.5$. At this forcing frequency, because of fundamental mode's simple shape, both DG and CLS can simulate the response quite well. The seventeenth natural frequency $f_{16}=50.038$ is associated with a 16-node mode-shape. At $f=50$, this shape amplifies and reduces in a beating fashion over a long time period, for the forcing frequency of $f=50$. Even though the motion is simple harmonic, DG can not resolve this complex mode-shape as well as it did for the first mode; which can be attributed to complex shape of the mode and relative proximity to the natural frequency. 


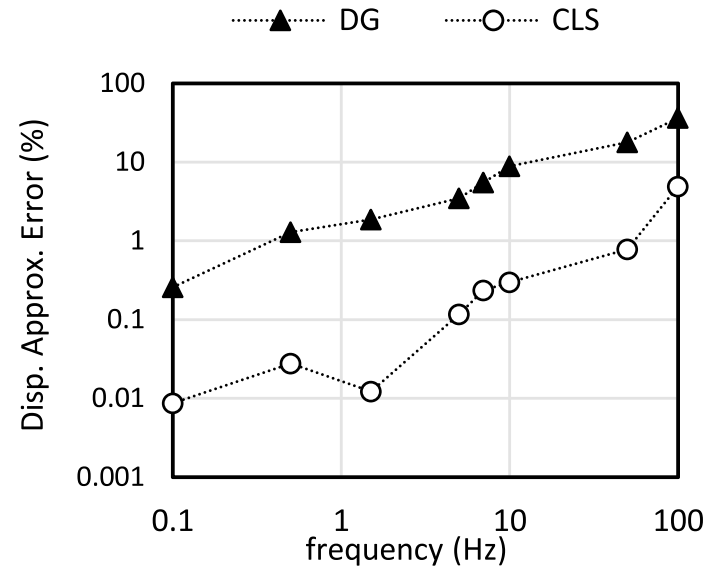

(a) Displacement error vs. forcing frequency

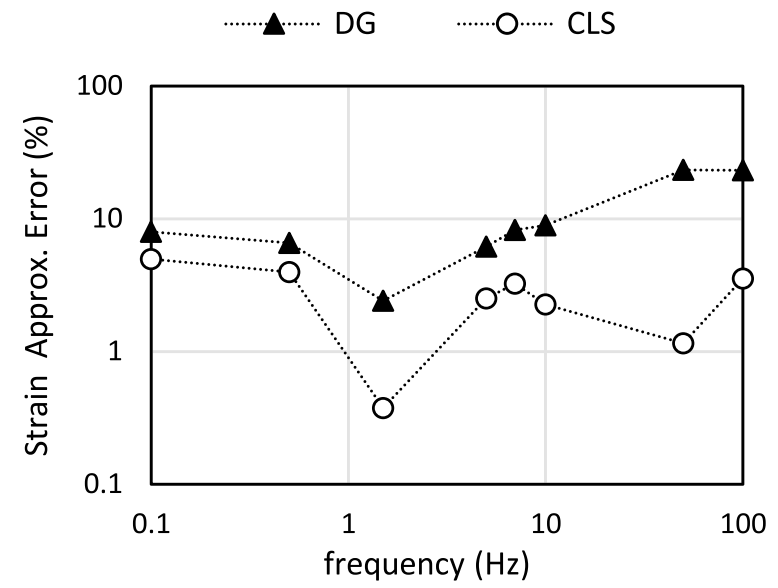

(b) Strain error vs. frequency

Fig. 4 Comparison of error in displacement and strain distributions as computed by Decoupled Galerkin (DG) and Coupled Least Squares (CLS) for different forcing frequencies

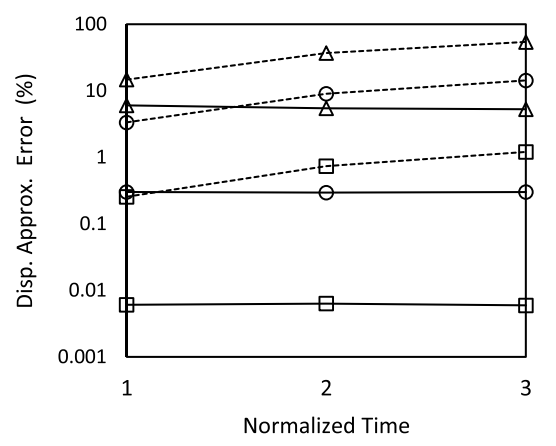

(a) Displacement error vs. long-span periods

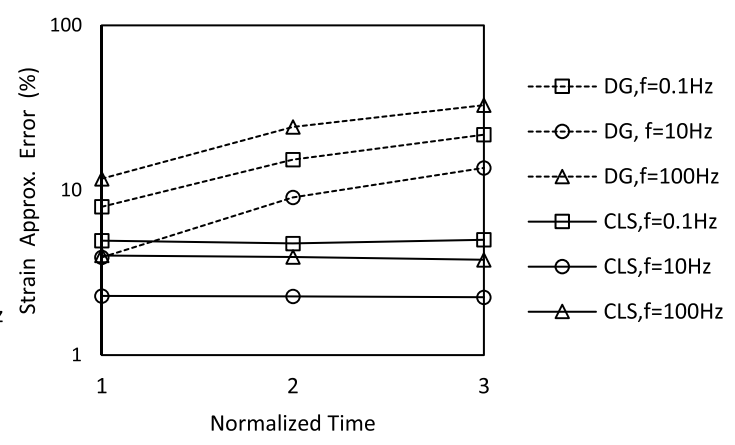

(b) Strain error vs. long-span periods

Fig. 5 Comparison of error in displacement and strain distributions as computed by DG and CLS for each of the first three long-span periods

\subsubsection{Evolution error}

In any finite element process, solution error depends on the variational form as well as the mesh and $p$-level utilized. A necessary characteristic of a viable time dependent computational framework is to be free of evolution-error; i.e. it should yield solutions for which the computational error does not increase with time. In order to test for existence of evolution-error in DG and CLS a normalized time scale is adopted; which indicates the evolution over the first three long-span periods of oscillation.

Since the problem is linear and undamped, complex interactions observed over small time segments are part of a periodic motion that repeats itself over longer time spans, duration of which depends on what the forcing frequency is and how close it might be to a natural frequency. The long-span periods for three forcing frequencies of $\{0.1,10,100\} \mathrm{Hz}$ were observed from solution graphs generated using the exact solution. DG and CLS solution errors were measured over each of the first three longspan periods separately, Fig. 5

As can be seen from Fig. 5, regardless of the forcing frequency value, error present in CLS remains unchanged over each of the three consecutive time intervals; contrarily, it increases in the DG framework, for all forcing frequencies. Error increase during the evolution can lead to a divergent process or yield seriously erroneous results; especially when multi-layer effects, damping, or state dependent material properties are considered.

\subsection{Axial wave propagation}

The second test-case investigates the presence of numerical diffusion in CLS an DG computational frameworks. The medium considered is a rod of unit area with unit length, $L=1 \mathrm{~m}$. Mass density and elastic modulus are 


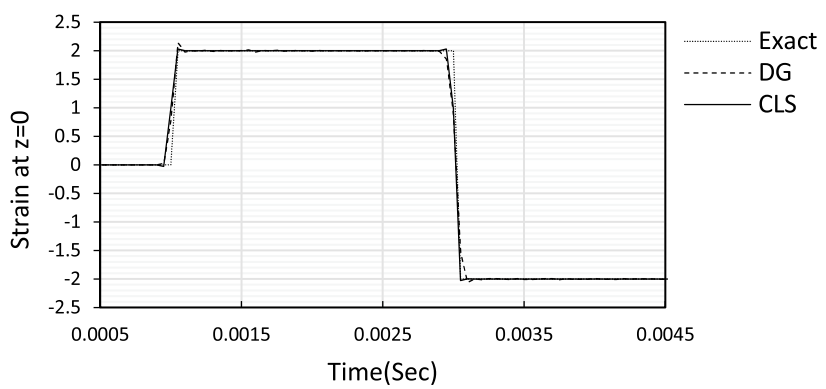

(a) Strain wave for $t \in[0.0005,0.0045]$ which includes $t_{0}=0.002$

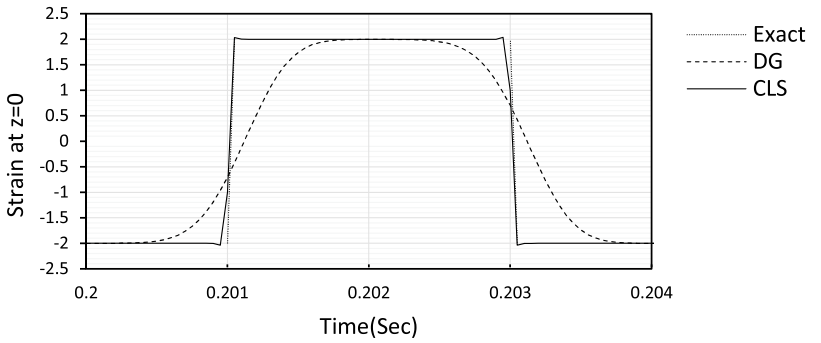

(b) Strain wave for $t \in[0.2,0.204]$

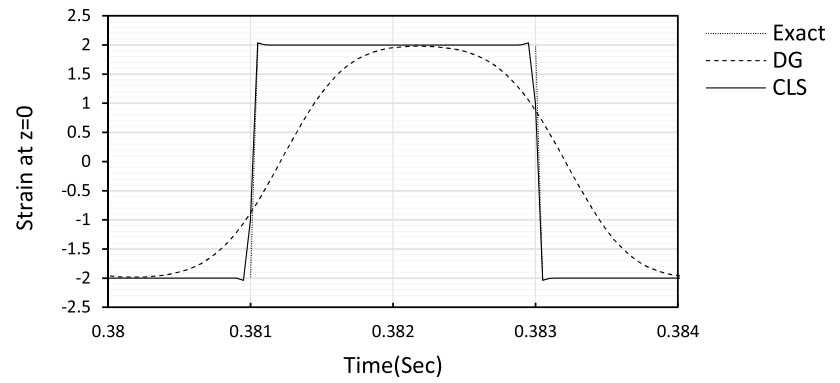

(c) Strain wave for $t \in[0.38,0.384]$

Fig. 6 Strain versus time over three time intervals; of duration $\Delta t=0.004 \mathrm{~s}$

$\rho=1000 \mathrm{~kg} / \mathrm{m}^{3}$ and $\lambda=E=1 \mathrm{GPa}$ respectively. The rod is fixed at $z=0$ and loaded with distributed force, $P$ at $z=1$.

Cases of constant loading, $P=P_{c}$ and single harmonic loading, $P=P_{h}$ are examined here. In each case the load is applied from $t=0$ to the time $t_{0}$ at which the reflective stress wave has reached its maximum value. Hence, the constant force $P_{c}=10^{9} \mathrm{GPa}$ is applied from $t=0$ to $t=t_{0}=0.002 \mathrm{~s}$; and the case of harmonic loading, $P_{h}=10^{9} \sin (2 \pi 100 t) \mathrm{GPa}$ is applied from zero to $t=t_{0}=0.003 \mathrm{~s}$. In both cases force is removed for $t>t_{0}$ allowing the rod to oscillate freely till $t=0.4 \mathrm{~s}$.

\subsubsection{Exact solution}

Closed form solution to (1) based on boundary conditions stated here, will be,

$$
\begin{aligned}
u(z, t)= & \frac{-8 L P}{E \pi^{2}} \sum_{k=1,3, \ldots m}^{\infty} \frac{1}{k^{2}} \\
& {\left[\sin \left(\frac{k \pi}{2}\right) \sin \left(\frac{k \pi z}{2 L}\right) \cos \left(\frac{k \pi c t}{2 L}\right)\right]+\frac{P z}{E} }
\end{aligned}
$$

\subsubsection{Numerical diffusion}

In a vibrating system, a uniform increase in wave's base is the effect of numerical diffusion present in the computational process. In order to separate the error due to numerical diffusion from that of numerical dispersion, highly refined temporal meshing is used to smooth out dispersion effects. Strain wave evolution at the fixed boundary, due to application of constant force $P_{c}$, is shown over three different time spans (Fig. 6). Figure 6a shows the solution around the time of commencing the free vibration stage; note that there are almost no dispersive or diffusive numerical errors present in the solution.

Figure $6 \mathrm{~b}, \mathrm{c}$ compare the solutions after 0.2 and $0.38 \mathrm{~s}$ of free vibration. As can be observed, the increase in strain wave's base is sever in DG framework. Closer inspection also reveals that as DG solution evolves the maximum peak appears later in time, implying a reduction in wave speed as well.

Ratio of the area under computed strain curves, for a given wave cycle, to that predicted by the exact solution can be used to measure the error due to numerical diffusion. These calculations were carried for CLS and DG for both types of loading. The results are shown in Fig. 7 in which the horizontal axis is normalized time, $\hat{t}=\frac{t}{t_{f}}$ where $_{f}=0.4 \mathrm{~s}$.

\section{Discussion}

Simulation of wave propagation through solid medium, especially due to impact, is a computational challenge. As demonstrated herein, the commonly used Galerkin weak 


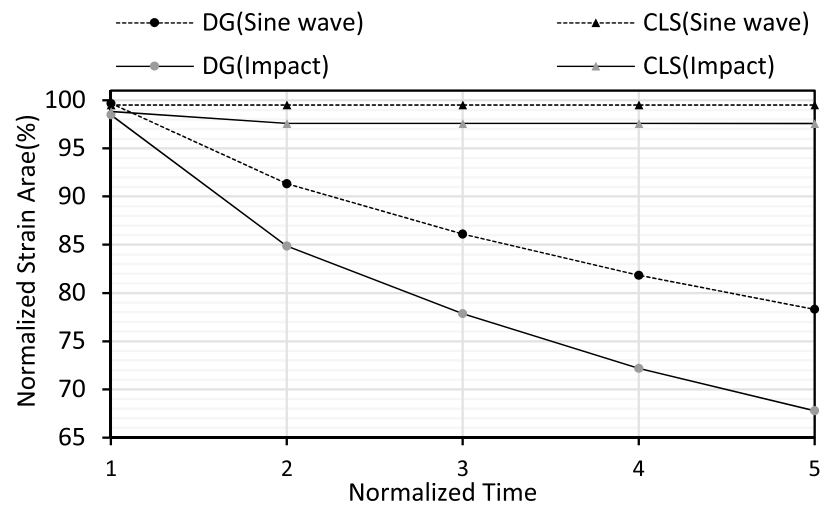

Fig. 7 Relative area under strain pulse as computed for CLS and DG

form of (1) cast over coupled or decoupled space-time domain, is not variationally-consistent because $\mathbb{C}^{0}$ elements can not provide a solution space in which up to the second derivatives $u$ are square integrable. Consequently, these forms require numerical stabilizers for a convergent evolutionary process. In contrast, second derivatives of $u$ are square integrable in CLS framework. Furthermore, CLS is not problem dependent, since it does not require any stabilizing parameter or process.

As demonstrated through numerical experiments, errors due to numerical dispersion, numerical diffusion, and accumulation over time are significantly lower in CLS framework. Furthermore, the finite elements used in CLS are of hierarchical type; yielding stable high order interpolations. High order interpolations are essential for a successful CLS process. This would not be attainable using standard finite elements, because the condition of resulting matrices deteriorates rapidly with increasing order of interpolation. Furthermore, since the choice of time increment in the evolution process is dictated by spatial element's size via relation (5), and the fact that high order interpolations allow for coarse spatial meshing, then larger time increments are possible when high order elements are used; and of course, coarser time meshing means lesser number of steps before reaching the final time in the evolution.

In all tests conducted, all necessary resources were utilized to obtain the most accurate results possible from each method. Overall, the run-time for DG took between three to four times longer than that of the comparable CLS.

\section{Conclusion}

Scope of this research was to investigate the viability and merits of the variationally-consistent CLS framework in studying wave propagation through solid type media.
This framework includes higher-continuity hierarchical finite elements as an inseparable part of it. CLS form of the wave equation was examined using $\mathbb{C}^{1}$ hierarchical finite elements. Here, it was shown that using a mesh of only a few elements, one can arbitrarily drive down computational errors through $p$-level increase. The advantage large-sized elements is the ability to take larger time steps. It was also shown that the time steps can be taken even longer and obtain a faster computation time if each time step is meshed.

In comparison to decoupled Galerkin formulation, $D G$, the least squares form, CLS, consistently yields lower errors; in some cases up to two orders of magnitude lower. Furthermore, it was shown that solution computed through CLS framework does not degrade over time; whereas the error in solutions obtained form decoupled Galerkin, which is the method used in all commercial finite element packages, consistently grows in time. Most importantly, CLS has virtually no numerical diffusion, this is in contrast to DG for which its inherent numerical diffusion causes a reduction of about $\% 40$ in stress wave's amplitude.

The space-time coupled least squares in conjunction with high $p$-level hierarchical $\mathbb{C}^{1}$ finite elements provide a robust and stable computational framework that can accurately simulate wave propagation through a solid medium. High accuracy and non-degrading character of the evolving solution, make this framework a reliable venue for simulating nonlinear effects; for which, lack of such properties can lead to highly erroneous results.

In conclusion, CLS framework is robust and time accurate with low accumulative errors, and low errors due to numerical dispersion and numerical diffusion.

\section{Compliance with ethical standards}

Conflict of interest The authors declare that they have no conflict of interest.

\section{References}

1. Kramer SL (2001) Geotechnical earthquake engineering, Prentice-Hall Internationals Series in Civil Engineering and Engineering Mechanics. Prentice-Hall, Upper Saddle River

2. Fornberg B (1996) A practical guide to pseudospectral methods. Cambridge University Press, Cambridge

3. Gustafsson B (2008) High order difference methods for time dependent PDE. Springer, Berlin

4. Shojaei A, Mossaiby F, Zaccariotto M, Galvanetto U (2017) The meshless finite point method for transient elastodynamic problems. Acta Mech 228:3581-3593

5. Hulbert GM, Hughes TJR (1990) Space-time finite element methods for second order hyperbolic equations. Comput Methods Appl Mech Eng 84:327-348 
6. Mirzaei D, Hasanpour K (2016) Direct meshless local PetrovGalerkin method for elastodynamic analysis. Acta Mech 227:519-632

7. Lisha H, Mohammed S (2016) A Runge-Kutta-Chebyshev SPH algorithm for elastodynamics. Acta Mech 227:1813-1835

8. Saffarian MA, Bagheripour MH (2014) Seismic response analysis of layered soils considering effect of surcharge mass using HFTD approach Part I: basic formulation and linear HFTD. Geomech Eng J 6:517-530

9. Saffarian MA, Bagheripour MH (2014) Seismic response analysis of layered soils considering effect of surcharge mass using HFTD approach Part II: nonlinear HFTD and numerical examples. Geomech Eng J 6:531-544

10. Sun C, Chung C (2008) Assessment of site effect of a shallow and wide basin using geotechnical information-based spatial characterization. Soil Dyn Earthq Eng 28:1028-1044

11. Hilber HM (1976) Analysis and design of numerical integration methods in structural dynamics. , Ph.D. Thesis, Earthquake Engineering Research Center, University of California, Berkeley

12. Hilber HM, Hughes TJR (1978) Collocation, dissipation and overshoot for time integration schemes in structural dynamics. Earthq Eng Struct Dyn 6:99-118

13. Hughes TJR (1983) Analysis of transient algorithms with particular reference to stability behavior. Computational methods for transient analysis (A 84-29160 12-64), 67-155

14. Hughes TJR (1987) The finite element method: linear static and dynamic finite element analysis. Prentice-Hall, Englewood Cliffs

15. Hilber HM, Hughes TJR, Taylor RL (1997) Improved numerical dissipation for time integration algorithms in structural dynamics. Earthq Eng Struct Dyn 5:283-292

16. Argyris JH, Scharpf DW (1969) Finite element in time and space. Nucl Eng Des 10:456-464

17. Fried I (1969) Finite element analysis of time-dependent phenomena. AIAA J 7:1170-1173

18. Oden JT (1969) A general theory of finite elements II. Applications. Int J Numer Methods Eng 1:247-259

19. Wilson EL, Nickell RE (1966) Application of finite element method to heat conduction analysis. Nucl Eng Des 4:1-11

20. Zienkiewicz OC (1977) The finite element method. McGraw-Hill, London

21. Zienkiewicz OC (1977) A new look at the Newmark, Houbolt and other time stepping formulas: a weighted residual approach. Earthq Eng Struct Dyn 5:413-418

22. Zienkiewicz OC, Parekh CJ (1977) Transient field problems-two and three dimensional analysis by isoparametric finite elements. Earthq Eng Struct Dyn 5:413-418

23. Varoglu E, Finn WDL (1980) Space-time finite elements incorporating characteristics for the Burgers equation. Int J Numer Methods Eng 16:171-184

24. Nguyen M, Reynen J (1984) A space-time least-square finite element scheme for advection-diffusion equations. Comput Methods Appl Mech Eng 42:331-342

25. Lewis DL, Lund J, Bowers KL (1987) The space-time Sinc-Galerkin method for parabolic problems. Int J Numer Methods Eng 24:1629-1644

26. Peters DA, Izadpanah AP (1988) Hp-version finite elements for the space-time domain. Comput Mech 3:73-88

27. Bruch JC, Zyvoloski G (1974) Transient two-dimensional heat conduction problems solved by the finite element method. Int J Numer Methods Eng 8:481-494
28. Morandi Cecchi M, Cella A (1973) A Ritz-Galerkin approach to heat conduction: method and results. In: Proceedings of the fourth Canadian congress of applied mechanics. Session $\mathrm{H}, \mathrm{pp}$ 767-768

29. Cheung YK, Tham LG (1979) Time-space finite elements for unsaturated flow through porous media. In: Proceedings of the third international conference on numerical methods in geomechanics, vol 1. A. A. Balkema, Rotterdam, pp 251-256

30. Bonnerot $R$, Jamet $P$ (1974) A second order finite element method for the one-dimensional Stefan problem. Int J Numer Methods Eng 8:811-820

31. Reed WH, Hill TR (1973) Triangular mesh methods for the neutron transport equation. Report LA-UR-73-479. Los Alamos Scientific Laboratory, Los Alamos

32. Hughes TJR, Hulbert GM (1988) Space-time finite element methods for elastodynamics: formulations and error estimates. Comput Methods Appl Mech Eng 66:339-363

33. Guo P, Wen-Hua W, Zhi-Gang W (2014) A time discontinuous Galerkin finite element method for generalized thermo-elastic wave analysis, considering non-Fourier effects. Acta Mech 25:299-307

34. Izadpanah E, Shojaee S, Hamzehei-Javaran S (2018) A timedependent discontinuous Galerkin finite element approach in two-dimensional elastodynamic problems based on spherical Hankel element framework. Acta Mech 229:4977-4994

35. Bell BC, Surana KS (1994) A space-time coupled p-version leastsquares finite element formulation for unsteady fluid dynamics problems. https://doi.org/10.1002/nme.1620372008

36. Szabo BA (1979) Some recent developments in finite element analysis. https://doi.org/10.1016/0898-1221(79)90063-4

37. Surana KS, Petti SR, Ahmadi AR, Reddy JN (2001) On p-version hierarchical interpolation functions for higher-order continuity finite element models. Int J Comput Eng Sci 2(4):653-673

38. Ahmadi AR (2003) Investigation of Galerkin and least squares finite element processes in higher order spaces. Ph.D. dissertation, University of Kansas, Lawrence, KS, US

39. Ahmadi AR, Surana KS, Maduri RK, Romkes A, Reddy JN (2009) Higher order global differentiability local approximations for 2-D distorted quadrilateral elements. Int J Comput Methods Eng Sci Mech 10:1-19

40. Surana KS, Ahmadi AR, Reddy JN (2002) The k-version of finite element method for self-adjoint operators in BVP. Int J Comput Eng Sci 3(2):155-218

41. Surana KS, Ahmadi AR, Reddy JN (2003) The k-version of finite element method for non-self-adjoint operators in BVP. Int J Comput Eng Sci 3(4):737-812

42. Surana KS, Ahmadi AR, Reddy JN (2004) The k-version of finite element method for nonlinear operators in BVP. Int J Comput Eng Sci 5(1):133-207

Publisher's Note Springer Nature remains neutral with regard to jurisdictional claims in published maps and institutional affiliations. 